\title{
Structural basis of transcription-translation coupling and collision in bacteria
}

\author{
Michael William Webster 1,2,3,4*, Maria Takacs ${ }^{1,2,3,4 *}$, Chengjin Zhu ${ }^{1,2,3,4}$, Vita Vidmar, ${ }^{1,2,3,4}$, Ayesha Eduljee ${ }^{1,2,3,4}$, \\ Mo'men Abdelkareem ${ }^{1,2,3,4}$, Albert Weixlbaumer ${ }^{1,2,3,4} \uparrow$
}

${ }^{1}$ Department of Integrated Structural Biology, Institut de Génétique et de Biologie Moléculaire et Cellulaire (IGBMC), 67404 IIIkirch, France. ${ }^{2}$ Université de Strasbourg, 67404 IIlkirch, France. ${ }^{3}$ CNRS UMR7104, 67404 Illkirch, France. ${ }^{4}$ INSERM U1258, 67404 Illkirch, France.

*These authors contributed equally to this work.

†Corresponding author. Email: albert.weixlbaumer@igbmc.fr

Prokaryotic messenger RNAs (mRNAs) are translated as they are transcribed. The pioneering ribosome potentially contacts RNA polymerase (RNAP), forming a supramolecular complex known as the expressome. The basis of expressome assembly and its consequences for transcription and translation are poorly understood. Here we present a series of structures representing uncoupled, coupled and collided expressome states determined by electron cryomicroscopy. A bridge between the ribosome and RNAP can be formed by the transcription factor NusG, stabilizing an otherwise variable interaction interface. Shortening of the intervening mRNA causes a substantial rearrangement that aligns the ribosome entrance-channel to the RNAP exit-channel. In this collided complex, NusG-linkage is no longer possible. These structures reveal mechanisms of coordination between transcription and translation and provide a framework for future study.

All organisms express genetic information in two steps. Messenger RNAs (mRNAs) are transcribed from DNA by RNA polymerase (RNAP), and then translated by ribosomes to proteins. In prokaryotes, translation begins as the mRNA is synthesized, and the pioneering ribosome on an mRNA is spatially close to RNAP $(1,2)$. Coordination of transcription with translation regulates gene expression and prevents premature transcription termination $(3,4)$. The trailing ribosome inhibits RNAP backtracking, contributing to synchronization of transcription and translation rates in vivo and in vitro (5-7).

Coordination may also involve physical contacts between RNAP and the ribosome. The conserved transcription factor NusG binds RNAP through its N-terminal domain (NusGNTD), and ribosomal protein uS10 through its C-terminal domain (NusG-CTD) both in vitro and in vivo $(8,9)$. Formation of a NusG-mediated bridge by simultaneous binding has not yet been observed, and the consequences of physical coupling are unknown. RNAP and the ribosome also interact directly (10-12). A transcribing-translating 'expressome' complex formed by collision of ribosomes with stalled RNAP in an in vitro translation reaction was reconstructed at $7.6 \AA$ resolution (10). This architecture would not permit a NusG-mediated bridge.

We sought to structurally characterize mechanisms of physical transcription-translation coupling, and resolve the relationship between NusG and the collided expressome. Expressomes were assembled by sequential addition of purified
Escherichia coli (E. coli) components (70S ribosomes, tRNAs, RNAP and NusG) to a synthetic DNA-mRNA scaffold (fig. S1, A to C). An mRNA with 38 nucleotides separating the RNAP active site from the ribosomal P-site was chosen to imitate a state expected to precede collision (13).

A reconstruction of the expressome was obtained at $3.0 \AA$ nominal resolution by electron cryomicroscopy (cryo-EM) (Fig. 1A; fig. S1, D and E; and table S1). RNAP and ribosome do not adopt a single relative orientation within the expressome, and focused refinement was required to attain a reconstruction of RNAP at $3.8 \AA$ nominal resolution (Fig. 1A and fig. S2; see materials and methods). Refined atomic models collectively present the key steps of prokaryotic gene expression within a single molecular assembly (Fig. 1B).

Direct contacts between RNAP and the ribosome, if they occur, are not stable in this complex and the mRNA is the only consistent connection. We characterized the dynamics of the complex by plotting the range of RNAP positions relative to the ribosome using the angular assignments of particles from focused reconstructions (Fig. 1C and fig. S3A). RNAP is loosely restrained to a plane perpendicular to an axis connecting the RNAP mRNA exit-channel to the ribosomal mRNA entrance-channel (movie S1). Within this plane, RNAP rotates freely. Seven clusters represent a series of preferred relative orientations (Fig. $1 \mathrm{C}$ and fig. S3A).

RNAP and ribosome models were placed in reconstructions generated from particles in clusters 1-6, while a large fraction of cluster 7 is predicted to be incompatible with 
longer upstream DNA (fig. S3, B to F, and table S2; see materials and methods). Expressome models represent characteristic relative orientations for each cluster, and collectively suggest a continuous movement of RNAP relative to the ribosome surface involving substantial changes in both rotation $\left(\sim 280^{\circ}\right)$ and translation ( $\left.50 \AA\right)$ (Fig. 1D and movie S1). The closest domain of RNAP to the ribosome is the zinc finger of the $\beta^{\prime}$ subunit $\left(\beta^{\prime}-\mathrm{ZF}\right)$ in all models. In clusters $1-3, \beta^{\prime}-\mathrm{ZF}$ sits within a funnel-shaped depression between the head, body, and shoulder domains of the $30 \mathrm{~S}$ subunit bounded by ribosomal proteins uS3, uS4 and uS5. We estimate RNAP transits from cluster 1 through clusters 2-5 to reach positions exemplified by model 6. Here, the RNAP $\beta^{\prime}-\mathrm{ZF}$ is between uS3 and uS10 on the 30S head domain.

NusG-NTD is bound to RNAP in expressome cluster 6 , but not clusters 1 and 2 (Fig. 1E). We determined that a substantial fraction of the imaged particles lacked NusG due to dissociation during gradient purification (fig. S3G). Importantly, the predicted position of the NusG-CTD bound to uS10 $(8,9)$ is closest to the NusG-NTD bound to RNAP in cluster 6 .

An improved reconstruction of the NusG-coupled expressome was obtained from a new sample prepared with increased NusG occupancy (Fig. 2A and fig. S4, A and B; see materials and methods). Conformational heterogeneity of ribosome and RNAP was substantially reduced, but focused refinement was required to obtain well-resolved ribosome and RNAP reconstructions (3.4 $\AA$ and $7.6 \AA$ respectively) (fig. S4, $\mathrm{C}$ to $\mathrm{E}$, and table $\mathrm{S} 1$ ). Continuous density in the unfocused reconstruction confirmed NusG bridges RNAP and the ribosome (Fig. 2A). We constructed an atomic model of the NusGcoupled expressome by fitting and refining a ribosome model, and docking a published RNAP-NusG-NTD model consistent with our map (14) into their consensus positions in the unfocused reconstruction (Fig. 2B).

Additional density corresponding to the NusG-CTD bound to uS10 was identified on the ribosome, which otherwise closely resembled that of the uncoupled expressome. The NusG-CTD is a KOW domain, consisting of a five-stranded $\beta$ barrel. As in the isolated NusG-uS10 complex determined by NMR (8), strand $\beta 4$ of NusG aligns with strand $\beta 4$ of uS10, forming an extended intermolecular $\beta$-sheet (Fig. 2C). Yet NusG and uS10 are significantly closer in the expressome than in the isolated complex because NusG loops L1 (F141 and F144) and L2 (I164, F165, R167) insert into a hydrophobic pocket of uS10 that is enlarged by movement of helix $\alpha 2$ (Fig. $2 \mathrm{D}$ and fig. S5, A to D). F165 of NusG in particular is embedded within uS10. This accounts for its crucial role in binding uS10 identified by mutational studies (9). The altered position of NusG not only increases the area contacting uS10 but avoids clashing with neighboring ribosomal protein uS3 (Fig. 2D).

The NusG-CTD recruits Rho to terminate synthesis of untranslated mRNAs (15). In the NusG-coupled expressome, NusG binds uS10 with the same interface it binds Rho, suggesting the events are mutually exclusive (fig. S5E) (16). The structure of the expressome thereby explains how the trailing ribosome is sensed by NusG, and transcription termination is consequently reduced.

Binding of the NusG-NTD to RNAP suppresses backtracking by stabilizing the upstream DNA duplex $(14,17)$. In the expressome, space for the upstream DNA is further restricted by an extended channel formed by uS10 and NusG (fig. S5F). The interaction of the NusG-CTD with $\mathrm{uS10}$ is predicted to reduce dissociation of NusG-NTD from RNAP through increased avidity (18). The RNAP-NusG complex within the coupled expressome is likely stabilized by the trailing ribosome, and transcription elongation is consequently favored.

The mRNA exit-channel of RNAP is separated from the entrance-channel of the ribosome by $\sim 60 \AA$. Continuous electron density on the solvent side of uS3 allowed modeling of the intervening 12 mRNA nucleotides, completing the mRNA path from synthesis to decoding (Fig. 2E and fig. S6, A to C). The interpretability of the electron density varies considerably, however, and this model is considered one of an ensemble of mRNA conformations.

The RNAP mRNA exit-channel is adjacent to uS3 residues R72, K79 and K80, and clear electron density for mRNA in this region suggests a relatively stable contact. The path continues to four arginines immediately outside the ribosomal mRNA entrance-channel (R126, R127, R131 and R132) (fig. S6A). R131 and R132 were previously identified as imparting ribosomal helicase activity (19). The mRNA path in this region is close to, but different from, that observed previously in structures of mRNA-bound ribosomes (20) (fig. S6, D to F).

Binding of the nascent transcript by uS3 likely modulates secondary structure formation. Structured mRNAs can decrease translation rates (21), stabilize transcriptional pauses (e.g., the E. coli his-pause (22)) or induce transcription termination (23). While the ribosome can unwind mRNA secondary structure with basic residues in the mRNA entrancechannel (19), preventing mRNAs folding downstream likely aids translation efficiency. We propose that by positioning RNAP in line with an extended series of basic residues, NusG helps keep nascent mRNAs single-stranded and thereby enhances both transcription and translation efficiency.

No stable contacts are observed between the core subunits of RNAP and the ribosome in the NusG-coupled expressome. The relative position of RNAP and the ribosome varies between particles, albeit substantially less than the sample with partial NusG occupancy (fig. S4, A and B). Analysis of movement by multi-body refinement (24) reveals RNAP is constrained to avoid clashes between $\beta^{\prime}-\mathrm{ZF}$ and the cavity formed by uS3, uS10, NusG and helix 33 of 16S rRNA (h33) into which it is inserted (Fig. 2F and movie S2). RNAP is also 
flexibly tethered to the ribosome by NusG, with the length of the NusG linker (residues 117-125) varying in the range 14-30 A.

To test whether lengthening the intervening mRNA alters the architecture of the expressome, we imaged two samples with four additional mRNA nucleotides (42 in total) separating the RNAP active site from the ribosomal P-site (fig. S7A and table S1). Saturation with NusG increased particle frequencies resembling the NusG-coupled expressome with shorter mRNA, including density linking the complexes (fig. S7, B to E). Compared to shorter mRNA, more particles are observed arranged similarly to cluster 7 of the uncoupled expressome (Fig. 1C). This arrangement is termed TTC-C by [Wang et al.]. However, NusG-CTD is bound to uS10 only in cluster 6 but not cluster 7, indicating NusG couples in only one arrangement (fig. S7F).

The mRNA spanning the mRNA exit- and entrance-channels is in an extended conformation in the NusG-coupled expressome. To test if coupling by NusG is possible when the spanning mRNA is shorter, we obtained a reconstruction of a NusG-containing expressome with an mRNA shortened to 34 nucleotides between the ribosomal P-site and the RNAP active site (Fig. 3A, fig. S8, and table S1). A model was constructed as described for the coupled expressome (Fig. 3B).

RNAP is positioned close to the ribosome mRNA entrance-channel, more than $50 \AA$ from its location in the NusGcoupled expressome. Consistent with this change, RNAP still binds the NusG-NTD but is no longer tethered through the NusG-CTD to uS10 because the NusG linker (residues 117-125; maximum extension $\sim 30 \AA$ ) would need to span an $85-145 \AA$ distance. We determined the structure of an equivalent sample lacking NusG, and confirmed the position of RNAP is very similar (fig. S9A and table S1). The architecture is therefore not NusG-dependent, and is similar to particles from clusters 1 and 2 of the uncoupled expressome (fig. S12). We conclude that RNAP coupling to the ribosome through NusG requires the P-site to be more than 34 nucleotides from the $3^{\prime}$ end of the mRNA.

The rearrangement of RNAP and ribosome in our structure with shortened mRNA resembles the expressome formed by collision of translating ribosomes with stalled RNAP (RNAP backbone RMSD 3Å based on 16S rRNA superposition) (10) (fig. S10, A and B, and fig. S12). We therefore term this molecular state the 'collided expressome'. The previous reconstruction was resolved to $7.6 \AA$, and our improved model allowed us to define the interaction surfaces of RNAP and the ribosome in more detail.

Four regions are in close proximity: uS10 with the N-terminal domain of the RNAP $\alpha 1$ subunit, uS3 with RNAP subunits $\alpha 1$ and the $\beta$-flap domain, uS4 with $\beta^{\prime}$-ZF, and uS2 with RNAP $\omega$-subunit (Fig. 3, C and D, and fig. S9, B and C). However, density for the $\omega$-subunit is very weak suggesting partial or complete dissociation occurs upon collision. The contacts bury a total surface area of $\sim 3000 \AA^{2}$. Yet RNAP moves relative to the ribosome, albeit less than in the samples previously analyzed (fig. S8, B and C). The RNAP ribosome contacts are likely transient, and the contact area consequently varies. The observed RNAP-ribosome configuration allows striking structural complementarity between the molecular surfaces.

Rotation of RNAP relative to the ribosome beyond the observed position would cause steric clashes (Fig. 4A and fig. S11). We hypothesize that the architecture of the collided expressome is the product of structural complementarity and the energetically-favorable minimization of mRNA path length. To test this, we generated $\sim 18,000$ hypothetical expressome models representing an exhaustive search of RNAP rotations about the mRNA axis at a series of distances along it ( $2^{\circ}$ rotational step size, $0.5 \AA$ translational step size). After excluding clashing models, we found that the shortest mRNA path is achieved by the RNAP orientations observed by cryoEM (Fig. 4B). A simple model is therefore sufficient to explain the observed orientation of RNAP relative to the ribosome: when inserting into the mRNA entrance-channel cavity on the ribosome, RNAP adopts an orientation with structural complementarity so the intervening mRNA spans the shortest distance.

We sought to clarify whether expressome formation is driven by concurrent binding to the same mRNA, or if specific ribosome-RNAP contacts contribute. Co-purification of RNAP with ribosomes was substantially reduced when the mRNA did not support concurrent ribosome binding but RNAP lacking DNA or mRNA entirely ('RNAP-core') bound ribosomes more stably (Fig. 4C and fig. S10, C and D). This observation was previously thought to indicate expressome formation is not driven by a shared mRNA $(10,12)$.

To examine this, we imaged samples assembled without further purification and lacking nucleic acid scaffolds (RNAP-core-70S) by negative stain EM. No expressomes formed, suggesting their formation is driven by concurrent mRNA binding and direct interactions play minor roles. However, we observed at least two alternative RNAP binding sites (Fig. 4D). The sites can be described only approximately from this data, but one (site I) is consistent with an interaction with ribosomal protein uS2 observed in a core RNAP-30S complex (11). Saturation of ribosomes with ribosomal protein bS1, which has no effect on expressome formation (fig. S13A), abolished occupancy of site I without affecting the second site (site II). Addition of a nucleic acid scaffold containing just a short mRNA (minimal scaffold) abolished occupancy of site II only, while addition of both (short mRNA scaffold, bS1) abolished both (fig. S13). A potential biological role remains to be shown, but the existence of additional 70S-RNAP contact modes highlights the complexity of their interaction. 
Thus, the expressome is mRNA-linked and consequently dynamic. A level of structural independence may be required to accommodate internal movements that occur during the reaction cycle of each complex. Coupling by NusG restrains RNAP motions, and happens at variable RNAP ribosome distances (fig. S12), but not when they collide (Fig. 4E). Relative orientations of the two machineries change in prevalence as a function of their separation (fig. S12). Importantly, translation factor binding is compatible with all the observed RNAP orientations. The role of the presented structures in vivo remains to be investigated, and this study provides a basis for elucidating the role of coupling in gene expression, and its regulation by transcription factors and regulatory mRNA structures.

\section{REFERENCES AND NOTES}

1. R. Byrne, J. G. Levin, H. A. Bladen, M. W. Nirenberg, The in vitro formation of a DNA ribosome complex. Proc. Natl. Acad. Sci. U.S.A. 52, 140-148 (1964). doi:10.1073/pnas.52.1.140 Medline

2. O. L. Miller Jr., B. A. Hamkalo, C. A. Thomas Jr., Visualization of bacterial genes in action. Science 169, 392-395 (1970). doi:10.1126/science.169.3943.392 Medline

3. C. Yanofsky, Attenuation in the control of expression of bacterial operons. Nature 289, 751-758 (1981). doi:10.1038/289751a0 Medline

4. J. P. Richardson, Preventing the synthesis of unused transcripts by Rho factor. Cell 64, 1047-1049 (1991). doi:10.1016/0092-8674(91)90257-Y Medline

5. S. Proshkin, A. R. Rahmouni, A. Mironov, E. Nudler, Cooperation between translating ribosomes and RNA polymerase in transcription elongation. Science 328, 504508 (2010). doi:10.1126/science.1184939 Medline

6. M. Zhu, M. Mori, T. Hwa, X. Dai, Disruption of transcription-translation coordination in Escherichia coli leads to premature transcriptional termination. Nat. Microbiol. 4, 2347-2356 (2019). doi:10.1038/s41564-019-0543-1 Medline

7. F. Stevenson-Jones, J. Woodgate, D. Castro-Roa, N. Zenkin, Ribosome reactivates transcription by physically pushing RNA polymerase out of transcription arrest. Proc. Natl. Acad. Sci. U.S.A. 117, 8462-8467 (2020). doi:10.1073/pnas.1919985117 Medline

8. B. M. Burmann, K. Schweimer, X. Luo, M. C. Wahl, B. L. Stitt, M. E. Gottesman, P. Rösch, A NusE:NusG complex links transcription and translation. Science 328, 501-504 (2010). doi:10.1126/science.1184953 Medline

9. S. Saxena, K. K. Myka, R. Washburn, N. Costantino, D. L. Court, M. E. Gottesman, Escherichia coli transcription factor NusG binds to 70 S ribosomes. Mol. Microbiol. 108, 495-504 (2018). doi:10.1111/mmi.13953 Medline

10. R. Kohler, R. A. Mooney, D. J. Mills, R. Landick, P. Cramer, Architecture of a transcribing-translating expressome. Science 356, 194-197 (2017). doi:10.1126/science.aal3059 Medline

11. G. Demo, A. Rasouly, N. Vasilyev, V. Svetlov, A. B. Loveland, R. Diaz-Avalos, N. Grigorieff, E. Nudler, A. A. Korostelev, Structure of RNA polymerase bound to ribosomal 30S subunit. eLife 6, e28560 (2017). doi:10.7554/eLife.28560 Medline

12. H. Fan, A. B. Conn, P. B. Williams, S. Diggs, J. Hahm, H. B. Gamper Jr., Y.-M. Hou, S. E. O'Leary, Y. Wang, G. M. Blaha, Transcription-translation coupling: Direct interactions of RNA polymerase with ribosomes and ribosomal subunits. Nucleic Acids Res. 45, 11043-11055 (2017). doi:10.1093/nar/gkx719 Medline

13. D. Castro-Roa, N. Zenkin, In vitro experimental system for analysis of transcriptiontranslation coupling. Nucleic Acids Res. 40, e45 (2012). doi:10.1093/nar/gkr1262 Medline

14. J. Y. Kang, R. A. Mooney, Y. Nedialkov, J. Saba, T. V. Mishanina, I. Artsimovitch, R. Landick, S. A. Darst, Structural Basis for Transcript Elongation Control by NusG Family Universal Regulators. Cell 173, 1650-1662.e14 (2018). doi:10.1016/i.cell.2018.05.017 Medline

15. S. L. Sullivan, M. E. Gottesman, Requirement for E. coli NusG protein in factordependent transcription termination. Cell 68, 989-994 (1992). doi:10.1016/0092-8674(92)90041-A Medline

16. M. R. Lawson, W. Ma, M. J. Bellecourt, I. Artsimovitch, A. Martin, R. Landick, K.
Schulten, J. M. Berger, Mechanism for the Regulated Control of Bacterial Transcription Termination by a Universal Adaptor Protein. Mol. Cell 71, 911922.e4 (2018). doi:10.1016/imolcel.2018.07.014 Medline

17. M. Turtola, G. A. Belogurov, NusG inhibits RNA polymerase backtracking by stabilizing the minimal transcription bubble. elife 5, e18096 (2016). doi:10.7554/eLife.18096 Medline

18. G. Vauquelin, S. J. Charlton, Exploring avidity: Understanding the potential gains in functional affinity and target residence time of bivalent and heterobivalent ligands. Br. J. Pharmacol. 168, 1771-1785 (2013). doi:10.1111/bph.12106 Medline

19. S. Takyar, R. P. Hickerson, H. F. Noller, mRNA helicase activity of the ribosome Cell 120, 49-58 (2005). doi:10.1016/i.cell.2004.11.042 Medline

20. H. Amiri, H. F. Noller, Structural evidence for product stabilization by the ribosomal mRNA helicase. RNA 25, 364-375 (2019). doi:10.1261/rna.068965.118 Medline

21. X. Qu, J.-D. Wen, L. Lancaster, H. F. Noller, C. Bustamante, I. Tinoco Jr., The ribosome uses two active mechanisms to unwind messenger RNA during translation. Nature 475, 118-121 (2011). doi:10.1038/nature10126 Medline

22. C. L. Chan, R. Landick, The Salmonella typhimurium his operon leader region contains an RNA hairpin-dependent transcription pause site. Mechanistic implications of the effect on pausing of altered RNA hairpins. J. Biol. Chem. 264, 20796-20804 (1989). Medline

23. I. Gusarov, E. Nudler, The mechanism of intrinsic transcription termination. Mol. Cell 3, 495-504 (1999). doi:10.1016/S1097-2765(00)80477-3 Medline

24. T. Nakane, D. Kimanius, E. Lindahl, S. H. Scheres, Characterisation of molecular motions in cryo-EM single-particle data by multi-body refinement in RELION. eLife 7, e36861 (2018). doi:10.7554/eLife.36861 Medline

25. K. R. Andersen, N. C. Leksa, T. U. Schwartz, Optimized E. coli expression strain LOBSTR eliminates common contaminants from His-tag purification. Proteins 81, 1857-1861 (2013). doi:10.1002/prot.24364 Medline

26. K.-A. F. Twist, S. I. Husnain, J. D. Franke, D. Jain, E. A. Campbell, B. E. Nickels, M. S. Thomas, S. A. Darst, L. F. Westblade, A novel method for the production of in vivo-assembled, recombinant Escherichia coli RNA polymerase lacking the $\alpha \mathrm{C}$ terminal domain. Protein Sci. 20, 986-995 (2011). doi:10.1002/pro.622 Medline

27. Y. Zhang, U. Werling, W. Edelmann, SLiCE: A novel bacterial cell extract-based DNA cloning method. Nucleic Acids Res. 40, e55 (2012). doi:10.1093/nar/gkr1288 Medline

28. X. Guo, A. G. Myasnikov, J. Chen, C. Crucifix, G. Papai, M. Takacs, P. Schultz, A. Weixlbaumer, Structural Basis for NusA Stabilized Transcriptional Pausing. Mol. Cell 69, 816-827.e4 (2018). doi:10.1016/j.molcel.2018.02.008 Medline

29. M. N. Vassylyeva, J. Lee, S. I. Sekine, O. Laptenko, S. Kuramitsu, T. Shibata, Y. Inoue, S. Borukhov, D. G. Vassylyev, S. Yokoyama, Purification, crystallization and initial crystallographic analysis of RNA polymerase holoenzyme from Thermus thermophilus. Acta Cryst. D58, 1497-1500 (2002) doi:10.1107/S0907444902011770 Medline

30. D. Moazed, H. F. Noller, Transfer RNA shields specific nucleotides in 16 S ribosomal RNA from attack by chemical probes. Cell 47, 985-994 (1986). doi:10.1016/0092-8674(86)90813-5 Medline

31. G. Blaha, U. Stelzl, C. M. T. Spahn, R. K. Agrawal, J. Frank, K. H. Nierhaus, Preparation of functional ribosomal complexes and effect of buffer conditions on tRNA positions observed by cryoelectron microscopy. Methods Enzymol. 317, 292-309 (2000). doi:10.1016/S0076-6879(00)17021-1 Medline

32. A. R. Subramanian, Structure and functions of ribosomal protein S1. Prog. Nucleic Acid Res. Mol. Biol. 28, 101-142 (1983). doi:10.1016/S0079-6603(08)60085-9 Medline

33. R. Jünemann, J. Wadzack, F. J. Triana-Alonso, J.-U. Bittner, J. Caillet, T. Meinnel, K. Vanatalu, K. H. Nierhaus, In vivo deuteration of transfer RNAs: Overexpression and large-scale purification of deuterated specific tRNAs. Nucleic Acids Res. 24, 907-913 (1996). doi:10.1093/nar/24.5.907 Medline

34. E. Cayama, A. Yépez, F. Rotondo, E. Bandeira, A. C. Ferreras, F. J. Triana-Alonso, New chromatographic and biochemical strategies for quick preparative isolation of tRNA. Nucleic Acids Res. 28, e64 (2000). doi:10.1093/nar/28.12.e64 Medline

35. S. Q. Zheng, E. Palovcak, J.-P. Armache, K. A. Verba, Y. Cheng, D. A. Agard, MotionCor2: Anisotropic correction of beam-induced motion for improved cryoelectron microscopy. Nat. Methods 14, 331-332 (2017). doi:10.1038/nmeth.4193 Medline 
36. K. Zhang, Gctf: Real-time CTF determination and correction. J. Struct. Biol. 193, 1-12 (2016). doi:10.1016/j.jsb.2015.11.003 Medline

37. J. Zivanov, T. Nakane, B. O. Forsberg, D. Kimanius, W. J. H. Hagen, E. Lindahl, S. H. W. Scheres, New tools for automated high-resolution cryo-EM structure determination in RELION-3. elife 7, e42166 (2018). doi:10.7554/eLife.42166 Medline

38. D. Liebschner, P. V. Afonine, M. L. Baker, G. Bunkóczi, V. B. Chen, T. I. Croll, B. Hintze, L.-W. Hung, S. Jain, A. J. McCoy, N. W. Moriarty, R. D. Oeffner, B. K. Poon, M. G. Prisant, R. J. Read, J. S. Richardson, D. C. Richardson, M. D. Sammito, O. V. Sobolev, D. H. Stockwell, T. C. Terwilliger, A. G. Urzhumtsev, L. L. Videau, C. J. Williams, P. D. Adams, Macromolecular structure determination using X-rays, neutrons and electrons: Recent developments in Phenix. Acta Cryst. D75, 861877 (2019). doi:10.1107/S2059798319011471 Medline

39. J. Noeske, M. R. Wasserman, D. S. Terry, R. B. Altman, S. C. Blanchard, J. H. D. Cate, High-resolution structure of the Escherichia coli ribosome. Nat. Struct. Mol. Biol. 22, 336-341 (2015). doi:10.1038/nsmb.2994 Medline

40. J. Y. Kang, P. D. B. Olinares, J. Chen, E. A. Campbell, A. Mustaev, B. T. Chait, M. E. Gottesman, S. A. Darst, Structural basis of transcription arrest by coliphage HK022 Nun in an Escherichia coli RNA polymerase elongation complex. eLife 6 , e25478 (2017). doi:10.7554/eLife.25478 Medline

41. E. Schmitt, M. Panvert, S. Blanquet, Y. Mechulam, Crystal structure of methionyltRNAfMet transformylase complexed with the initiator formyl-methionyltRNAfMet. EMBO J. 17, 6819-6826 (1998). doi:10.1093/emboj/17.23.6819 Medline

42. R. T. Byrne, A. L. Konevega, M. V. Rodnina, A. A. Antson, The crystal structure of unmodified tRNAPhe from Escherichia coli. Nucleic Acids Res. 38, 4154-4162 (2010). doi:10.1093/nar/gka133 Medline

43. E. F. Pettersen, T. D. Goddard, C. C. Huang, G. S. Couch, D. M. Greenblatt, E. C. Meng, T. E. Ferrin, UCSF Chimera-A visualization system for exploratory research and analysis. J. Comput. Chem. 25, 1605-1612 (2004). doi:10.1002/icc.20084 Medline

44. M. Selmer, C. M. Dunham, F. V. Murphy 4th, A. Weixlbaumer, S. Petry, A. C. Kelley, J. R. Weir, V. Ramakrishnan, Structure of the 70S ribosome complexed with mRNA and tRNA. Science 313, 1935-1942 (2006). doi:10.1126/science.1131127 Medline

45. R. M. Voorhees, A. Weixlbaumer, D. Loakes, A. C. Kelley, V. Ramakrishnan, Insights into substrate stabilization from snapshots of the peptidyl transferase center of the intact 70 S ribosome. Nat. Struct. Mol. Biol. 16, 528-533 (2009). doi:10.1038/nsmb.1577 Medline

46. N. Fischer, P. Neumann, A. L. Konevega, L. V. Bock, R. Ficner, M. V. Rodnina, H. Stark, Structure of the E. coli ribosome-EF-Tu complex at $<3 \AA$ resolution by Cscorrected cryo-EM. Nature 520, 567-570 (2015). doi:10.1038/nature14275 Medline

47. N. R. James, A. Brown, Y. Gordiyenko, V. Ramakrishnan, Translational termination without a stop codon. Science 354, 1437-1440 (2016). doi:10.1126/science.aai9127 Medline

48. P. Emsley, K. Cowtan, Coot: Model-building tools for molecular graphics. Acta Cryst. D60, 2126-2132 (2004). doi:10.1107/S0907444904019158 Medline

49. T. I. Croll, ISOLDE: A physically realistic environment for model building into lowresolution electron-density maps. Acta Cryst. D74, 519-530 (2018). doi:10.1107/S2059798318002425 Medline

50. A. J. Jakobi, M. Wilmanns, C. Sachse, Model-based local density sharpening of cryo-EM maps. eLife 6, e27131 (2017). doi:10.7554/eLife.27131 Medline

51. J. B. Heymann, M. Chagoyen, D. M. Belnap, Common conventions for interchange and archiving of three-dimensional electron microscopy information in structural biology. J. Struct. Biol. 151, 196-207 (2005). doi:10.1016/i.jsb.2005.06.001 Medline

52. Y. Zhang, S. Hong, A. Ruangprasert, G. Skiniotis, C. M. Dunham, Alternative Mode of E-Site tRNA Binding in the Presence of a Downstream mRNA Stem Loop at the Entrance Channel. Structure 26, 437-445.e3 (2018). doi:10.1016/i.str.2018.01.013 Medline

53. A. B. Loveland, A. A. Korostelev, Structural dynamics of protein S1 on the 70S ribosome visualized by ensemble cryo-EM. Methods 137, 55-66 (2018). doi:10.1016/i.ymeth.2017.12.004 Medline

54. M. A. Lauber, J. Rappsilber, J. P. Reilly, Dynamics of ribosomal protein S1 on a bacterial ribosome with cross-linking and mass spectrometry. Mol. Cell.
Proteomics 11, 1965-1976 (2012). doi:10.1074/mcp.M112.019562 Medline

\section{ACKNOWLEDGMENTS}

We thank Julio Ortiz, Corinne Crucifix, Xieyang Guo and Tat Cheung Cheng for help with data collection at the IGBMC. We thank Wim Hagen and Felix Weis for help with data collection at the EMBL in Heidelberg, Germany. We thank Venki Ramakrishnan and Ann C. Kelley for their valuable help in ribosome and tRNA purification. This work has been supported by iNEXT PID 6979, funded by the Horizon 2020 program of the European Union. We acknowledge the European Synchrotron Radiation Facility for the provision of microscope time on CM01, and we thank Gregory Effantin and Michael Hons for their assistance. We thank members of the Weixlbaumer lab for critical reading of the manuscript. Funding: The authors were supported by the French Infrastructure for Integrated Structural Biology (FRISBI ANR-10-INBS-05, Instruct-ERIC, and grant ANR-10LABX-0030-INRT, a French State fund managed by the Agence Nationale de la Recherche under the program Investissements d'Avenir ANR-10-IDEX-000202). The work was supported by an EMBO long-term fellowship to M.W.W. and the ERC starting grant TRANSREG (679734) to A.W. Author contributions: M.W.W. and M.T. performed experiments, binding assays, electron microscopy, and data analysis. C.Z. participated in data analysis and purification of NusG. M.W.W., M.T., V.V., A.D.E., M.A. and A.W. built and refined atomic models. A.W. designed and supervised research. M.W.W., M.T., and A.W. prepared the manuscript with input from all authors. Competing interests: Authors declare no competing interests. Data and materials availability: Electron density maps for uncoupled, coupled, and collided expressomes (with and without NusG) were deposited in the EM database (EMD-11418, EMD-11419, EMD-11420, EMD-11421, EMD-11422, EMD-11423 and EMD-11426). Refined coordinates were deposited in the PDB database under accession codes 6ZTN (NusG-coupled expressome with RNA-42) 6ZT0, 6ZU1 and 6ZTP (models for uncoupled expressome clusters 1, 2, and NusG-coupled expressome cluster 6 with RNA-38), 6ZT J (NusG-coupled expressome with RNA-38), 6ZTL (collided expressome with NusG), and 6ZTM (collided expressome without NusG). Materials are available from the authors on request.

\section{SUPPLEMENTARY MATERIALS}

science.sciencemag.org/cgi/content/full/science.abb5036/DC1

Materials and Methods

Figs. S1 to S13

Tables S1 and S2

References (25-54)

MDAR Reproducibility Checklist

Movies S1 to S3

28 February 2020; accepted 17 July 2020

Published online 20 August 2020

10.1126/science.abb5036 

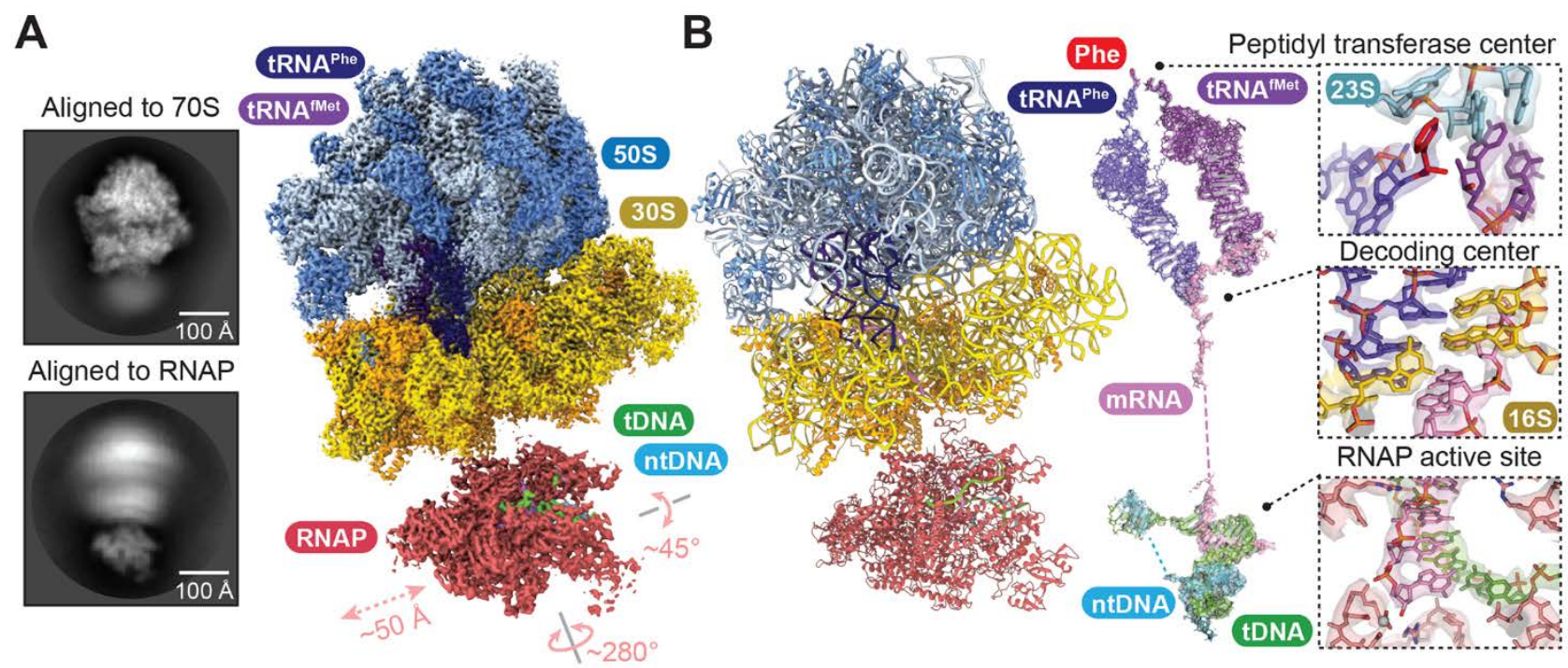

C

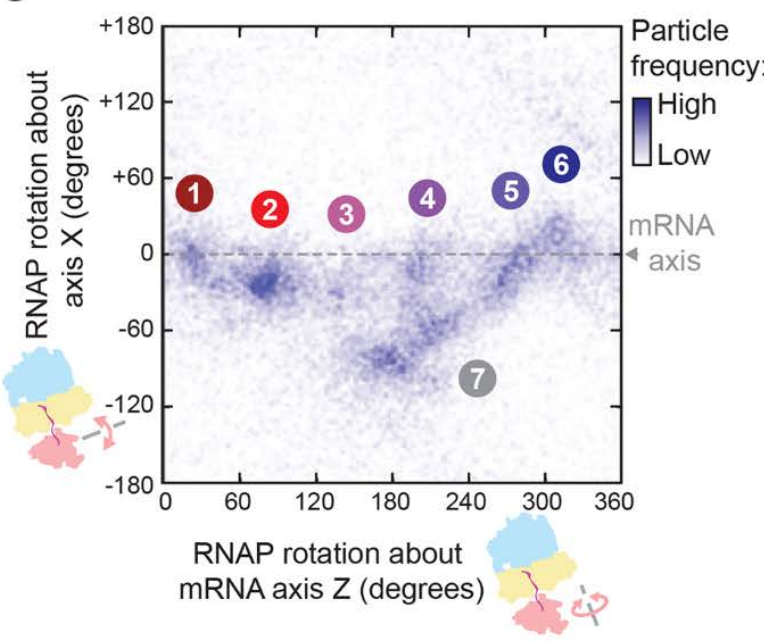

D

E

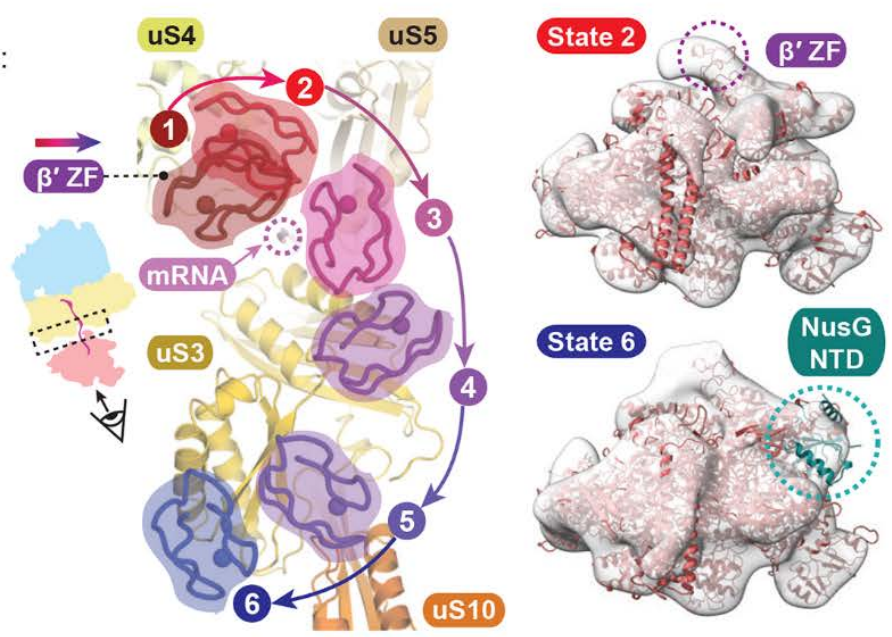

Fig. 1. Structural models of the uncoupled expressome. (A) Representative cryo-EM 2D class averages showing conformational variability (left), and cryo-EM maps of ribosome and RNAP in the uncoupled expressome (right). RNAP is shown in position 2 (see panel E), with measured rotation and translations of RNAP indicated. (B) Atomic model of the uncoupled expressome in ribbon representation (left), and the central steps in gene expression shown by segmented cryo-EM maps with superimposed atomic coordinates (right). (C) Plot of RNAP-70S relative orientation with clusters indicating a series of orientations (1-6) distinguished by rotation of RNAP. Further characterization of expressome particles resembling cluster 6 (Fig. 2), revealed these are likely physically coupled via NusG. Cluster 7 primarily includes particles with orientations incompatible with longer upstream DNA, but also states characterized by [Wang et al.]. (D) Representative positions of the RNAP $\beta^{\prime}-Z F$ in each expressome model relative to the ribosome surface. (E) NusG is present in state 6 (dashed green circle) but not in state 2. The position of $\beta^{\prime}-Z F$ is shown (purple dashed circle). Focused cryo-EM maps shown filtered to $20 \AA$ resolution with fitted coordinates. 


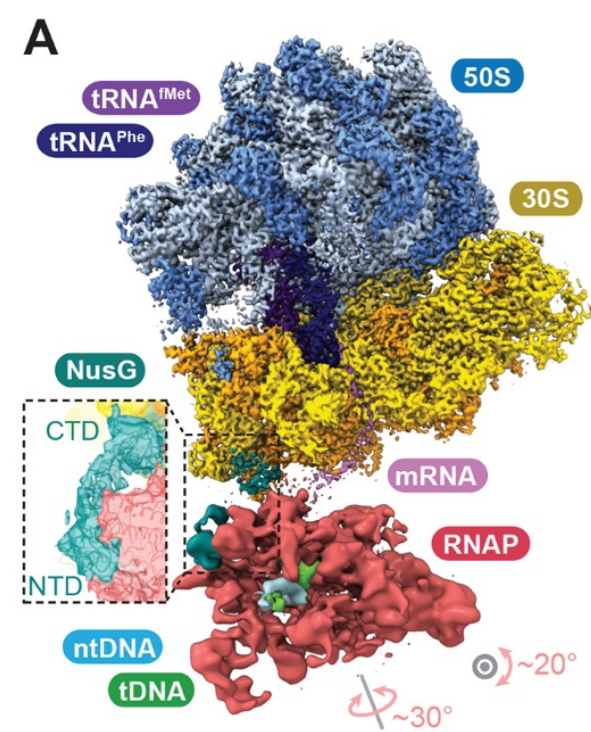

$\mathbf{E}$

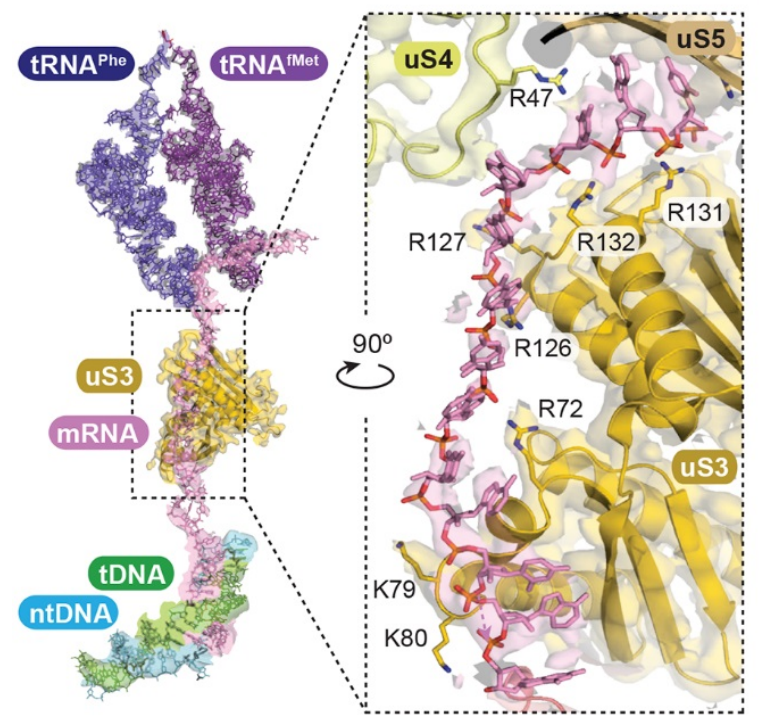

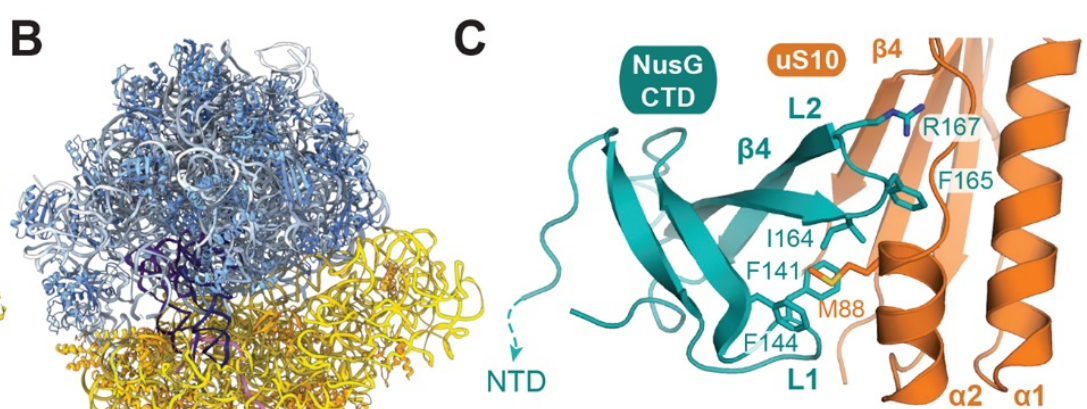

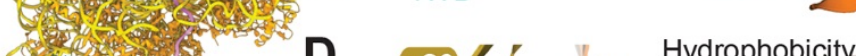

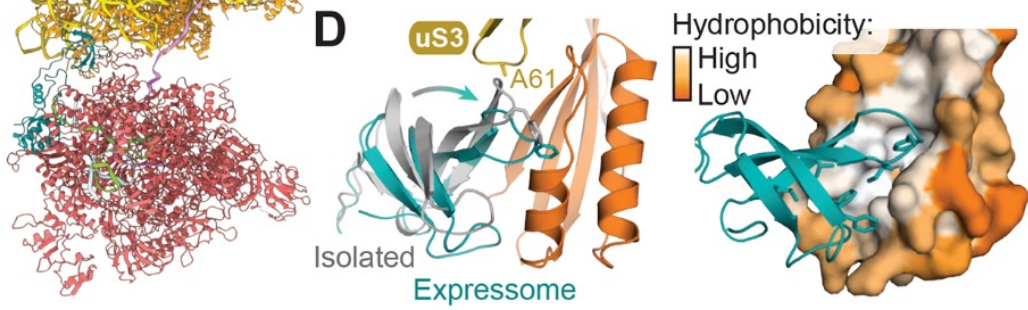

$\mathbf{F}$
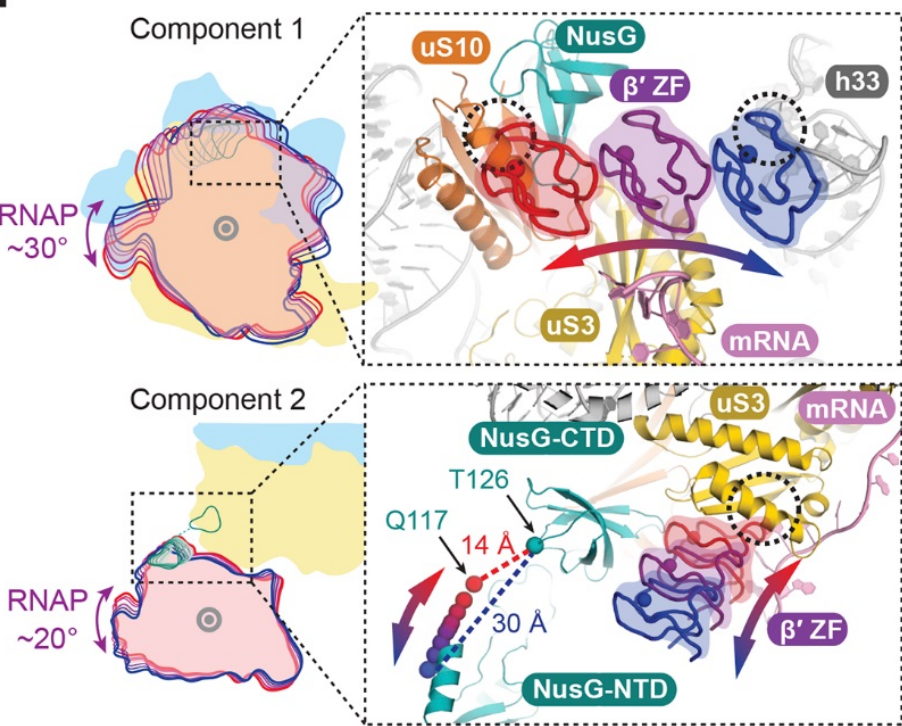

Fig. 2. Structural models of the NusG-coupled expressome. (A) Focused cryo-EM maps of ribosome and RNAP in the NusG-coupled expressome. Inset shows continuous electron density between NusGNTD and -CTD domains in unfocused map filtered to $8 \AA$ (slice view). (B) Ribbon representations of the NusG-coupled expressome model. (C) Interaction of NusG-CTD with ribosomal protein uS10. (D) Structural superposition with the isolated NusG-uS10 complex based on alignment to uS10 (grey; PDB code 2KVQ) (left), and hydrophobic pocket created by conformational change of uS10 (right). (E) mRNA connecting the ribosome mRNA entrance-channel to RNAP exit-channel shown by cryo-EM map filtered to $4 \AA$ and fitted model. (F) The range of RNAP positions relative to the ribosome surface determined by multi-body refinement. Cartoon of two principal components accounting for $44 \%$ of variance (left). Component 1 involves rotation in a plane approximately parallel to the surface of the ribosome and is limited by clashes between the $\beta^{\prime}$-ZF of RNAP and either US10 or h33 (dashed circles). Component 2 is an orthogonal rotation limited by extension of the flexible NusG linker (residues 117126) in one direction (red through purple to blue arrow; and clash between $\beta^{\prime}$-ZF and uS3 in the other (dashed circle). Positions of RNAP $\beta^{\prime}-Z F$ and NusG residue Q117 indicate trajectories (red through purple to blue). 

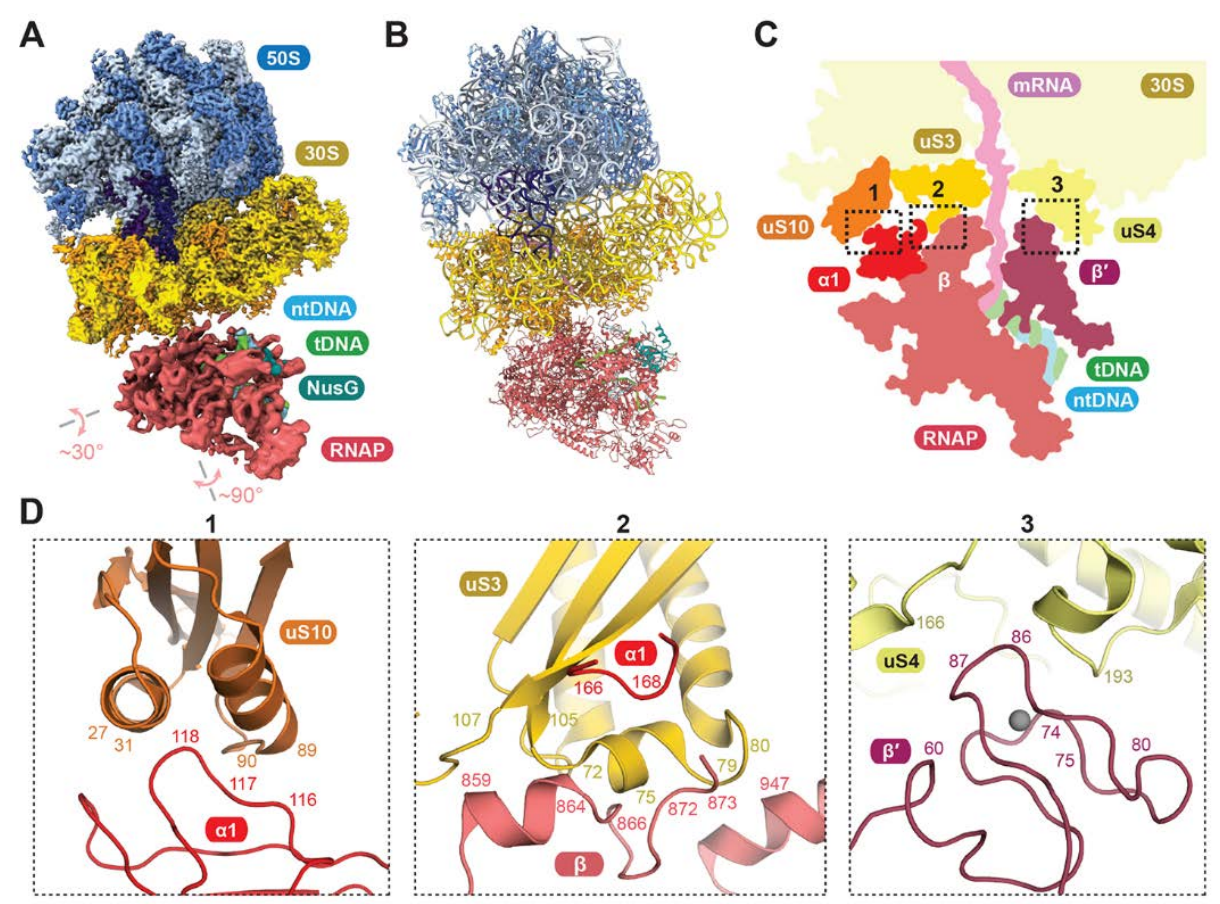

Fig. 3. Structural models of the collided expressome. (A and B) Cryo-EM maps and model of the collided expressome. (C) Schematic cross-section indicating three regions of close contact between RNAP and ribosome. (D) Details of the interaction interfaces of RNAP with ribosome. 

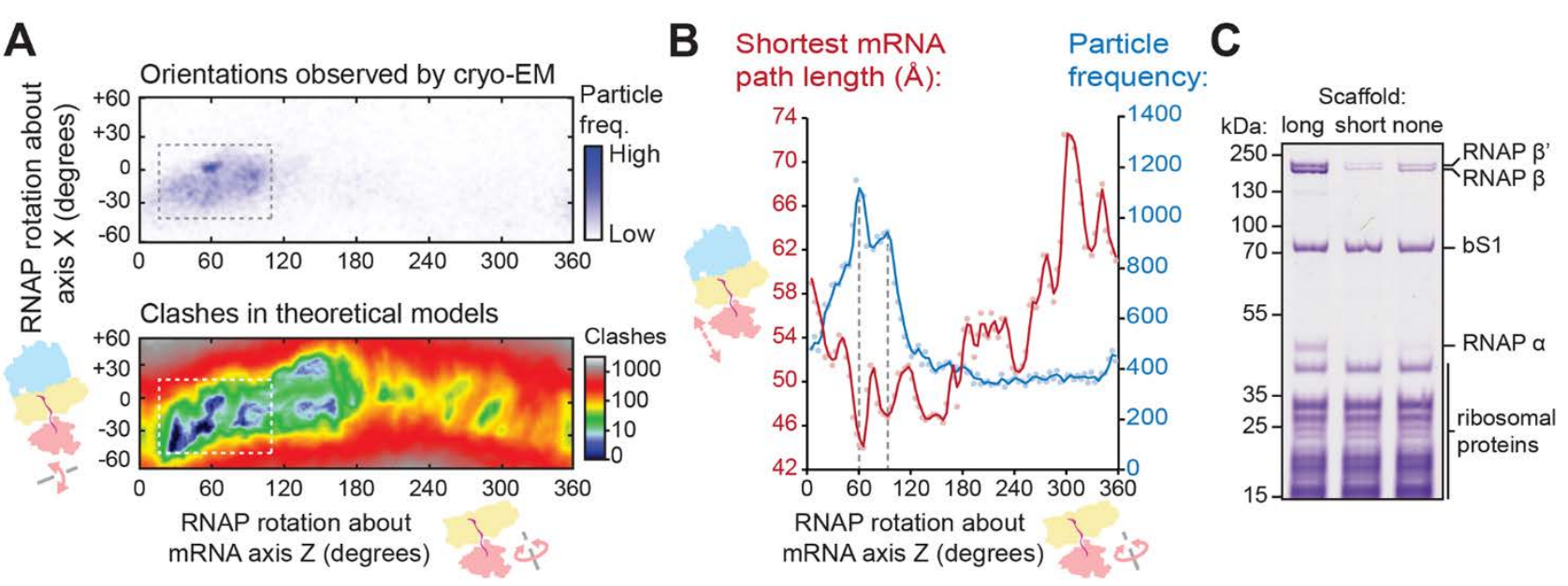

D

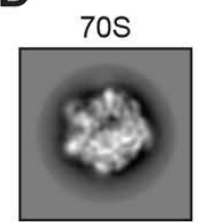

Expressome
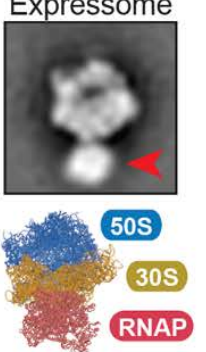

E

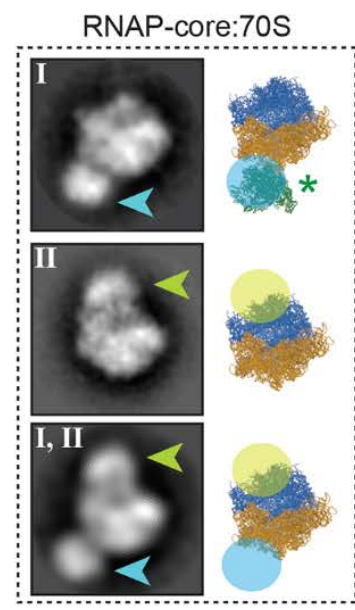

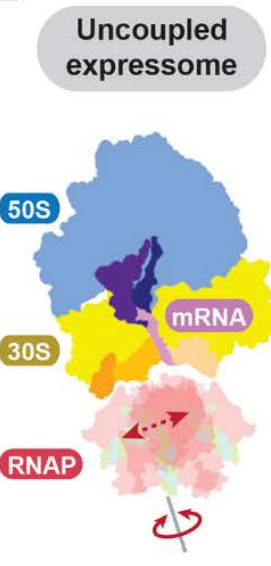

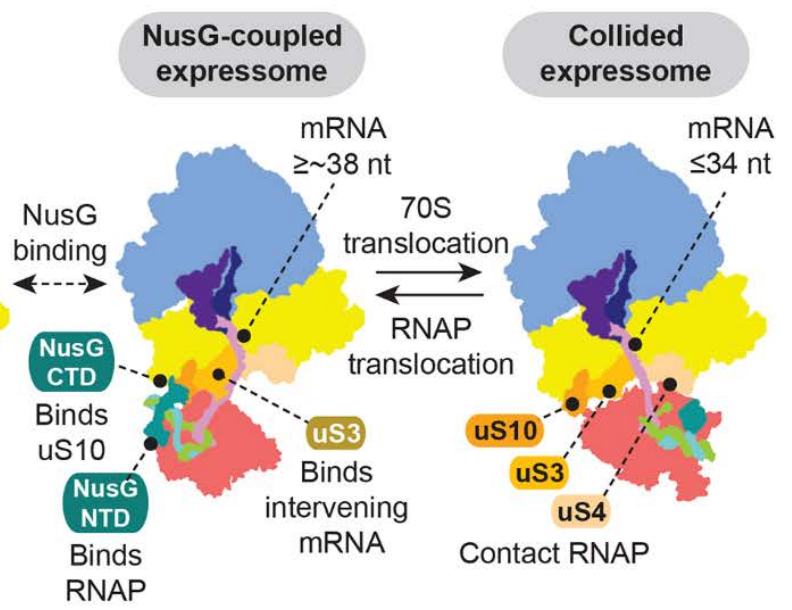

Fig. 4. Formation of RNAP-70S complexes. (A) Collided expressome RNAP-70S relative orientations observed by cryo-EM (top) correspond to a restricted space that avoids steric clashes (bottom). (B) The most common RNAP positions in the collided expressome (blue line) coincide with minima of the intervening mRNA path length (red line). (C) Gradient co-purification of RNAP with 70S ribosomes depends on the nucleic acid scaffold. RNAP-70S complexes were formed under low-salt conditions using an mRNA long enough to allow ribosome binding ('long'), or not ('short'), or no nucleic acids ('none'). Coomassie-stained SDS-PAGE of ribosome-containing sucrose gradient peak shown. (D) Negative stain EM class averages of 70S-RNAP complexes show distinct binding sites for core RNAP sample (cyan and lime arrows) compared with expressome sample (red arrow). Position of RNAP from 30S-RNAP complex superimposed (green asterisk). (E) Key features and interchange between expressome complexes during transcription-translation coordination. In the uncoupled expressome, RNAP is loosely restrained and adopts various orientations. Coupling by NusG aligns the mRNA with ribosomal protein US3 and restricts the position of RNAP. Once the ribosome approaches RNAP further, the collided state forms in which the mRNA length is limiting and NusG no longer links the two machineries. 